\title{
Insulin secretion and insulin resistance trajectories over 1 year after kidney transplantation: a multicenter prospective cohort study
}

\author{
Jun Bae Bang ${ }^{1}$, Su Hyung Lee ${ }^{1}$, Ja Young Jeon ${ }^{2}$, Chang-Kwon Oh ${ }^{1}$
}

${ }^{1}$ Division of Transplantation, Department of Surgery, Ajou University School of Medicine, Suwon, Korea
${ }^{2}$ Division of Endocrine, Department of Internal Medicine, Ajou University School of Medicine, Suwon, Korea

Background: We investigated the changing patterns of insulin secretion and resistance and risk factors contributing to the development of post-transplant diabetes mellitus (PTDM) in kidney recipients under tacrolimus-based immunosuppression regimen during 1 year after transplantation.

Methods: This was a multicenter prospective cohort study. Of the 168 subjects enrolled in this study, we analyzed a total 87 kidney transplant recipients without diabetes which was assessed by oral glucose tolerance test before transplantation. We evaluated the incidence of PTDM and followed up the index of insulin secretion (insulinogenic index [IGI]) and resistance (homeostatic model assessment for insulin resistance [HOMA-IR]) at 3 months, 6 months, 9 months, and 1 year after transplantation by oral glucose tolerance test and diabetes treatment. We also assessed the risk factors for incident PTDM.

Results: PTDM developed in 23 of 87 subjects (26.4\%) during 1 year after transplantation. More than half of total PTDM (56.5\%) occurred in the first 3 months after transplantation. During 1 year after transplantation, insulin resistance (HOMA-IR) was increased in both PTDM and no PTDM group. In no PTDM group, the increase in insulin secretory function to overcome insulin resistance was also observed. However, PTDM group showed no increase in insulin secretion function (IGI). Old age, status of prediabetes and episode of acute rejection were significantly associated with the development of PTDM.

Conclusions: In tacrolimus-based immunosuppressive drugs regimen, impaired insulin secretory function for reduced insulin sensitivity contributed to the development of PTDM than insulin resistance during 1 year after transplantation.

Corresponding author: Jun Bae Bang

E-mail: bjb425@gmail.com

(c) The Korean Society for Transplantation

This is an Open Access article distributed under the terms of the Creative Commons Attribution Non-Commercial License (http://creativecommons.org/licenses/by-nc/4.0/) which permits unrestricted non-commercial use, distribution, and reproduction in any medium, provided the original work is properly cited. 